\title{
HOT TOPICS FROM THE TEVATRON
}

\author{
D. GLENZINSKI \\ Fermilab, P.O. Box 500, Batavia, IL 60510, USA \\ E-mail: douglasg@fnal.gov
}

\begin{abstract}
The Tevatron Run-II began in March 2001. To date, both the CDF and D $\varnothing$ experiments have collected $1 \mathrm{fb}^{-1}$ of data each. The results obtained from this data set were summarized at this conference in 39 parallel session presentations covering a wide range of topics. I summarize the most important of those results here and comment on some of the prospects for the future.
\end{abstract}

Keywords: Tevatron; $B_{s}$ Oscillations; top-quark mass; higgs

\section{Introduction}

The Tevatron is a $p \bar{p}$ synchrotron collider at Fermilab operating at a center-of-mass energy of $1.96 \mathrm{TeV}$. There are two collision points each instrumented with a general purpose $4 \pi$ detector (the CDF and D $\varnothing$ experiments). The first high statistics data $\left(\approx 100 \mathrm{pb}^{-1}\right.$ per experiment) was collected in Run-I from 1990-1995 at $\sqrt{s}=1.8 \mathrm{TeV}$ and culminated in the discovery of the top quark. The experiments and collider complex all then undertook major upgrades and Run-II began delivering steady data in about June 2001 with initial luminosities somewhat below the best luminosities achieved at the end of Run-I. Since then the accelerator performance has steadily improved. The data set collected by each experiment has doubled in size in each of the last 3 years and is expected to do so again in 2006. At the time of this conference each experiment had collected about $1000 \mathrm{pb}^{-1}$ (or $1 \mathrm{fb}^{-1}$ ) of data and the majority of the results presented at this conference used this full data set. For comparison, the results shown at ICHEP04 were using about $200 \mathrm{pb}^{-1}$ of data. Run-II is scheduled to last until the fall of 2009 and to deliver a total of $6-8 \mathrm{fb}^{-1}$ of data to each experiment.

\section{The Experiments}

Interested readers can find more detailed descriptions of the $\mathrm{CDF}^{1}$ and $\mathrm{D} \emptyset^{2}$ detectors in the references provided. Both are general purpose $4 \pi$ detectors centered on the $p \bar{p}$ beamline and collision point, azimuthally and forward-backward symmetric. They both feature precision silicon vertex detectors at the smallest radii, charged-particle tracking at intermediate radii, a superconducting solenoid which together with the tracking detectors allows the determination of charged-particle momentum in the plane transverse to the beamline $\left(p_{T}\right)$, calorimeters with electromagnetic and hadronic compartments, and finally, at the largest radii, muon chambers. While the Tevatron collision rate is about $2 \mathrm{MHz}$, only a small fraction of these collisions include high energy $p \bar{p}$ interactions. A multi-level on-line trigger is used to identify these high-energy interactions and data are recorded at a rate of about $100 \mathrm{~Hz}$ per experiment.

\section{Physics Program}

The Tevatron physics program is an extremely broad one, significantly contributing to the understanding of QCD, Electroweak, B-quark, and top-quark physics as well as significantly extending the sensitivities of searches for new physics arising from a wide 
variety of models. So far in Run-II CDF and $\mathrm{D} \emptyset$ have each published about 100 papers. In 2006 there was on average 1 Tevatron physics result published every six days. At this conference the Tevatron results were presented across 39 different parallel session talks ${ }^{3}$ and summarized across four different plenary talks ${ }^{4}$. Rather than attempt to summarize the full breadth of results, I concentrate here on those which are unique to the Tevatron physics program and which I consider the most important in terms of their impact. There are three: $B_{s}^{0}-\bar{B}_{s}^{0}$ oscillation results, the top-quark mass determination, and the search for the Standard Model (SM) higgs boson. These are among the most important physics topics being addressed at Run-II and I briefly summarize the status of each in the following sections.

\section{1. $B_{s}^{0}-\bar{B}_{s}^{0}$ Oscillations}

In the SM $B_{s}^{0}\left(\bar{B}_{s}^{0}\right)$ mesons can change into their anti-particle $\bar{B}_{s}^{0}\left(B_{s}^{0}\right)$ via higher-order electroweak box diagrams. The rate at which these $B_{s}^{0}-\bar{B}_{s}^{0}$ oscillations occur is proportional to the mass difference between the heavy and light flavor eigenstates, $\Delta m_{s}=$ $m\left(B_{s}^{H}\right)-m\left(B_{s}^{L}\right)$. A precision determination of $\Delta m_{s}$ would provide an important consistency check of the CKM quark-mixing matrix since $V_{t s} \sim \Delta m_{s}$.

The probability that a $B_{s}^{0}$ at proper time $t=0$ decays as a $\bar{B}_{s}^{0}$ at some later time, $t$, is given by $P($ mixed $)=\frac{1}{2} \Gamma \exp ^{-\Gamma t}(1-$ $\left.\cos \left(\Delta m_{s} t\right)\right)$, where $\frac{1}{\Gamma}=\tau$, the $B_{s}^{0}$ lifetime. The probability that the $B_{s}^{0}$ decays as a $B_{s}^{0}$ is similar, $P($ unmixed $)=\frac{1}{2} \Gamma \exp ^{-\Gamma t}(1+$ $\left.\cos \left(\Delta m_{s} t\right)\right)$. Experimentally, $\Delta m_{s}$ is extracted by constructing a time dependent asymmetry,

$$
A(t)=\frac{N_{+}(t)-N_{-}(t)}{N_{+}(t)+N_{-}(t)}
$$

where $N_{+(-)}(t)$ are the numbers of unmixed (mixed) events at time $t$ and are related to the above probability expressions. For a perfect detector the asymmetry is equivalent to a cosine curve of amplitude $1, A(t)=$ $\cos \left(\Delta m_{s} t\right)$. So $\Delta m_{s}$ can be determined by fitting for the frequency of the resulting asymmetry curve. In reality, experimental limitations, efficiencies, and resolutions all act to partially washout the asymmetry resulting in an extremely challenging measurement.

To determine the asymmetry and measure $\Delta m_{s}$ we need to determine 1) the flavor of the $B_{s}$ meson at production (ie. whether it a $B_{s}^{0}$ or $\bar{B}_{s}^{0}$ at $\left.\left.t=0\right), 2\right)$ the flavor of the $B_{s}$ meson at its decay, - by comparing the flavor at production and decay we can classify the event as mixed or unmixed - and 3) the proper decay time. Each of these is briefly discussed below. More detailed descriptions of these $B_{s}^{0}-\bar{B}_{s}^{0}$ mixing results can be found in Refs. ${ }^{5}$ and ${ }^{6}$.

The analysis begins by first reconstructing samples of $B_{s}$ meson decays. Both $\mathrm{CDF}$ and $\mathrm{D} \emptyset$ use the semi-leptonic decays ${ }^{7}$ $B_{s}^{0} \rightarrow D_{s}^{-} e^{+} \nu, D_{s}^{-} \mu^{+} \nu$, with the $D_{s}^{-}$reconstructed through $D_{s}^{-} \rightarrow \Phi \pi^{-} \rightarrow K^{+} K^{-} \pi^{-}$, $D_{s}^{-} \rightarrow K^{*} K^{-} \rightarrow K^{+} \pi^{-} K^{-}$, and $D_{s}^{-} \rightarrow$ $\pi^{+} \pi^{-} \pi^{-}$. Using $1 \mathrm{fb}^{-1}$ of data D $\varnothing$ collected about 36,500 semi-leptonic decays mostly in the $B_{s}^{0} \rightarrow D_{s}^{-} \mu^{+} \nu, D_{s}^{-} \rightarrow \Phi \pi^{-}$channel. Using all the above decay channels and $1 \mathrm{fb}^{-1}$ of data CDF collected about 37,000 semi-leptonic decays. The reconstructed $D_{s}^{-}$ mass for the semileptonic channel is shown in Figs. 1 and 2 for $\mathrm{D} \emptyset$ and $\mathrm{CDF}$, respectively. CDF also reconstructs $B_{s}$ decays in these fully-reconstructed hadronic channels, $B_{s}^{0} \rightarrow D_{s}^{-} \pi^{+}$and $B_{s}^{0} \rightarrow D_{s}^{-} \pi^{+} \pi^{-} \pi^{+}$using all three of the $D_{s}^{-}$decays described above. In $1 \mathrm{fb}^{-1}$ of data CDF collects about 3,600 fully-reconstructed hadronic decays. The mass distribution for these hadronic modes is shown in Fig. 3. In all channels the decay flavor is unambiguously determined from the charge of the associated $D_{s}^{ \pm}$meson. 


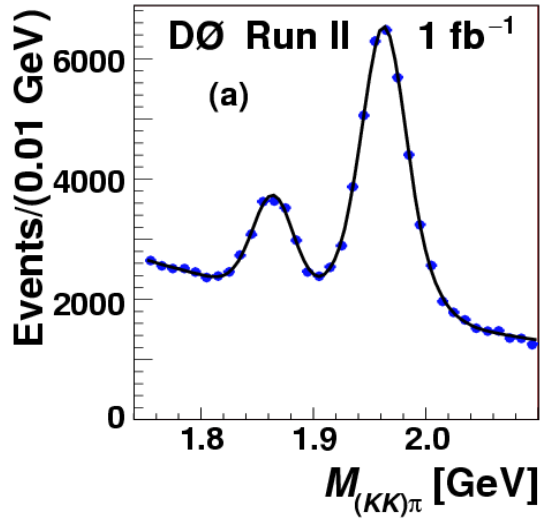

Fig. 1. The mass distribution for the $D_{s}$ candidates in semi-leptonic $B_{s}^{0}$ decays from $\mathrm{D} \varnothing$.

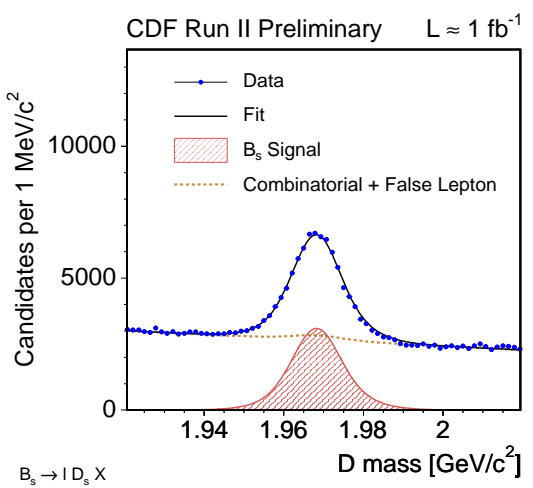

Fig. 2. The mass distributions for the $D_{s}$ candidates in semi-leptonic $B_{s}^{0}$ decays from CDF.

The tracks used to reconstruct the final state $B_{s}$ are constrained to originate from a common vertex and the proper time is determined as $t=M_{B_{s}} L_{T} / p_{T}^{B_{s}}$ where $M_{B_{s}}$ is the world-average mass of the $B_{s}$ meson, $p_{T}^{B_{s}}$ is the $p_{T}$ of the reconstructed $B_{s}^{0}$ meson estimated from the associated tracks used to reconstruct the $B_{s}^{0}$, and $L_{T}$ is the distance from the primary $p \bar{p}$ vertex to the $B_{s}^{0}$ vertex projected along $p_{T}^{B_{s}}$. For the hadronic decays, since they're fully reconstructed, $p_{T}^{B_{s}}=p_{T}^{t r k}$, while for the semi-leptonic decays, due to the missing neutrino, a correction is necessary, $p_{T}^{B_{s}}=p_{T}^{t r k} / \kappa$. A detailed Monte Carlo is used to determine $\kappa$. In the analysis the

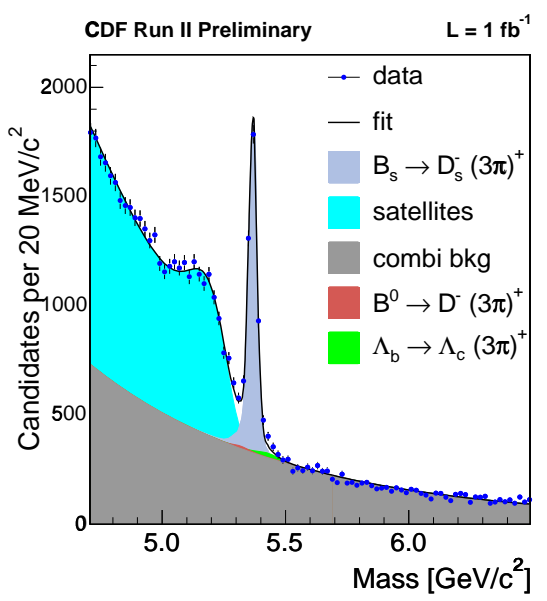

Fig. 3. The mass distribution for the fully reconstructed hadronic $B_{s}^{0}$ decays from CDF.

proper decay time is determine by integrating over $\kappa$ so that the rms of the $\kappa$ distribution effectively dilutes the proper time resolution of the semi-leptonic decays. By binning the semi-leptonic decays in lepton$D_{s}$ mass $B_{s}$ candidates with little missing momentum carry more weight in the final fit since their $\kappa$ distribution is quite narrow (and their proper time resolution much better). The average proper time resolution (at $\mathrm{t}=0$ ) for hadronic decays is about $87 \mathrm{fs}$, while the average proper time resolution for semi-leptonic decays varies from about $100 \mathrm{fs}$ for candidates with lepton- $D_{s}$ mass equal to $5.2 \mathrm{GeV} / c^{2}$ to about $200 \mathrm{fs}$ for candidates with lepton- $D_{s}$ mass equal to $3.0 \mathrm{GeV} / c^{2}$.

The production flavor is determined for each candidate using a suite of "flavor tagging" algorithms. The effectiveness of the flavor taggers is characterized by the quantity $\epsilon D^{2}$, where $\epsilon$ is the fraction of signal events with a tag and $D$ is the dilution and is related to $w$, the probability that the tagger decision is wrong, by $D=1-2 w$. Opposite-side flavor taggers take advantage of the fact that at the Tevatron b-quarks are predominantly produced in $b \bar{b}$ pairs. Both $\mathrm{CDF}$ and $\mathrm{D} \varnothing$ use lepton-charge ( $e$ and $\mu)$ and jet-charge opposite-side taggers. The performance of 
these opposite-side taggers is determined using large data control samples of $B^{+}$and $B^{0}$ mesons. The combined performance of all opposite-side taggers is $\epsilon D^{2}=1.5 \%$ for CDF and $2.5 \%$ for $\mathrm{D} \emptyset$. CDF also employs sameside taggers, which infer the production flavor from the charge of associated fragmentation tracks near the reconstructed $B_{s}^{0}$ candidate. The performance of the same-side taggers is determined from a detailed Monte Carlo sample of $B_{s}^{0}$ mesons which has been extensively tuned using large data control samples of $B^{+}$and $B^{0}$ decays. The performance of CDF's same-side tagger is $\epsilon D^{2}=$ $3.5 \%$, which, together with their oppositeside taggers gives them a total flavor-tagging performance of about $\epsilon D^{2}=5 \%$.

An unbinned maximum likelihood fit is performed to search for $B_{s}^{0}-\bar{B}_{s}^{0}$ oscillations. The likelihood combines mass, decay time, decay time resolution, and flavor tagging information event-by-event and includes components for signal and background contributions. Details can be found in Refs. ${ }^{5}$, ${ }^{8}$. A fit for the oscillation amplitude is performed as described in Ref. ${ }^{9}$ and behaves as a Fourier transform. For an assumed value of $\Delta m_{s}$ the oscillation amplitude is determined and a wide range scan of $\Delta m_{s}$ is considered. These "amplitude scans" yield an amplitude of $A=1$ at the true value of $\Delta m_{s}$ and $A=0$ otherwise.

At a given value of $\Delta m_{s}$ the sensitivity is given by $\sqrt{N_{B} \epsilon D^{2} / 2} \sqrt{\text { Purity }} e^{-\left(\Delta m_{s} \delta_{t} / 2\right)}$,

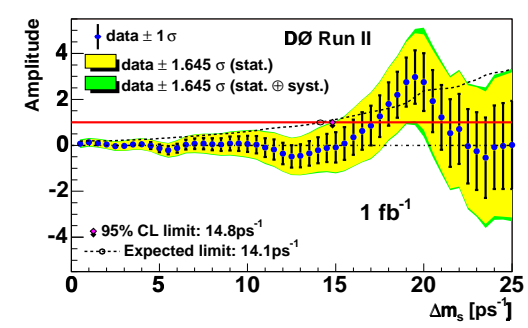

Fig. 4. The amplitude scan for the $\mathrm{D} \varnothing B_{s}^{0}-\bar{B}_{s}^{0}$ oscillation analysis. where $N_{B}$ is the number of reconstructed $B_{s}$ mesons, $\epsilon D^{2}$ is the total flavor-tagging performance, and $\delta_{t}$ is the proper time resolution. From this equation it's easy to understand that owing to the large sample size, the semi-leptonic decays are most important at small values of $\Delta m_{s}$ (less than about $10 \mathrm{ps}^{-1}$ ), while the hadronic decays, owing to their superior proper decay time resolution, dominate the sensitivity at large values of $\Delta m_{s}$ (greater than about $14 p s^{-1}$ ). The sensitivity for a given experiment is characterized by the expected $95 \%$ C.L. lower limit on $\Delta m_{s}$. In January 2006 the World-combined sensitivity, excluding the CDF and D $\varnothing$ results described here, was $18 \mathrm{ps}^{-1}$. The sensitivity of the $\mathrm{D} \emptyset$ analysis presented at this conference is $17 \mathrm{ps}^{-1}$ alone. The sensitivity of the CDF analysis presented at this conference is $26 p s^{-1}$ alone. CDF's superior sensitivity is due to their use of fully reconstructed hadronic decays and their use of same-side flavor taggers.

The $\mathrm{D} \emptyset$ amplitude scan is shown in Fig. 4. At $\Delta m_{s} \approx 19 \mathrm{ps}^{-1}$ the amplitude is consistent with one and deviates from zero. The probability that random tags could mimic a signal at least as significant is about $5 \%$. They use this result to set a two-sided limit of $17<\Delta m_{s}<21 p^{-1}$ at $90 \%$ C.L. ${ }^{5}$.

The CDF amplitude scan is shown in Fig. 5. At $\Delta m_{s} \approx 17 p s^{-1}$ the ampli-

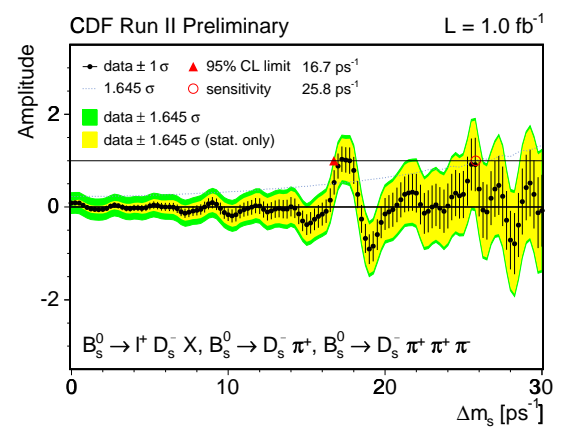

Fig. 5. The amplitude scan for the CDF $B_{s}^{0}-\bar{B}_{s}^{0}$ oscillation analysis. 


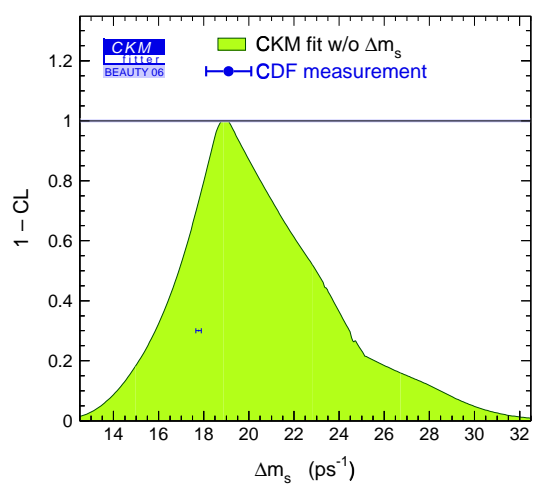

Fig. 6. A comparison of the measured $\Delta m_{s}$ to the region allowed by the indirect constraints using the rest of the CKM inputs.

tude is consistent with one and deviates from zero. The probability that random tags could mimic a signal at least as significant is about $0.2 \%$. Assuming this signal originates from real $B_{s}^{0}-\bar{B}_{s}^{0}$ oscillations, they determine $\Delta m_{s}=17.31_{-0.18}^{+0.33}$ (sta.) \pm 0.07 (sys.) $\mathrm{ps}^{-1}{ }^{6}$, which is compared to the indirect constraints on $\Delta m_{s}$ using the rest of the CKM inputs in Fig. $6{ }^{13},{ }^{14}$. This determination of $\Delta m_{s}$ is limited by statistical uncertainties. This is used to derive the ratio $\left|V_{t d} / V_{t s}\right|=\xi \sqrt{\frac{\Delta m_{d}}{\Delta m_{s}} \frac{M_{B_{s}}}{M_{B_{d}}}}$, where $\Delta m_{d}=0.505 \pm 0.005 \mathrm{ps}^{-1}{ }^{10}, \xi=$ $1.21_{-0.035}^{+0.04711}$, and $M_{B_{s}} / M_{B_{d}}=0.98390$ with negligible uncertainty ${ }^{12}$. Using their measured value of $\Delta m_{s}$ CDF finds $\left|V_{t d} / V_{t s}\right|=$ $0.208_{-0.002}^{+0.001}$ (exp.) ${ }_{-0.006}^{+0.008}$ (theo.). A comparison of this measurement with the indirect constraints from the rest of the CKM inputs is shown in Fig. $7{ }^{13},{ }^{14}$. This determination is approximately five times more precise than the previous determination and is limited by the Lattice QCD uncertainties on $\xi$.

The CDF collaboration has recently reported an improved $B_{s}^{0}-\bar{B}_{s}^{0}$ oscillation analysis which yields a signal significance in excess of $5 \sigma^{15}$. The measured value of $\Delta m_{s}$ and resulting $\left|V_{t d} / V_{t s}\right|$ are consistent with the values reported here.

A precision determination of $\Delta m_{s}$ also

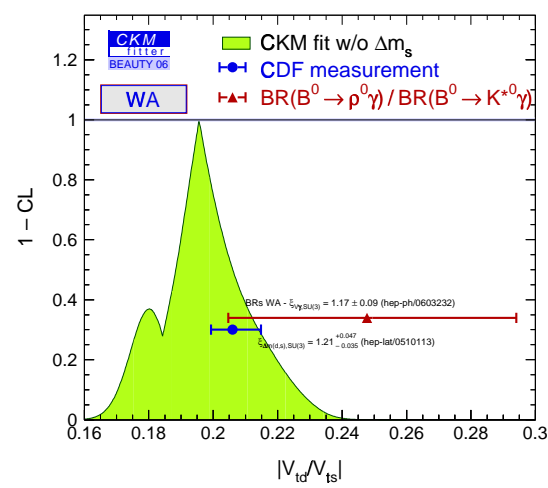

Fig. 7. A comparison of measurements of $\left|V_{t d} / V_{t s}\right|$ to indirect constraints from the rest of the CKM inputs.

allows a probe of new physics by parameterizing $\Delta m_{s}=C_{N P} \Delta m_{s}^{S M}$ and fitting for $C_{N P}$, where $\Delta m_{s}^{S M}$ is the Standard Model value for $\Delta m_{s}$. It's important to note that new physics can affect $\Delta m_{s}$ such that $C_{N P}$ can be either $>1$ or $<1$. New physics can also affect the CP phase in the $B_{s}$ sector and its contributions are parameterized as $\Phi_{B_{s}}=\Phi_{B_{s}}^{S M}+\Phi^{N P}$. For the $B_{s}$ the SM predicted CP phase is only $\Phi_{B_{s}}^{S M} \sim 1^{\circ}$ (cf. $\Phi_{B_{d}}^{S M} \sim 20^{\circ}$ ), so even small new physics effects should readily manifest themselves. The $\mathrm{D} \emptyset$ collaboration reported the first constraints on $\Phi_{B_{s}}$ by combining results from 3 analyses ${ }^{16}$ as shown in Fig. 8. Using CDF's $\Delta m_{s}$ result, $\mathrm{D} \emptyset$ ś $\Phi_{B_{s}}$ result, and the indirect determination of $\Delta m_{s}^{S M}$ from the rest of the CKM inputs yields constraints in the $\left(C_{N P}, \Phi^{N P}\right)$ plane as shown in Fig. $9^{17}$. All the measurements affecting these constraints are limited by statistical uncertainties and should continue to improve as CDF and D $\varnothing$ accumulate larger data sets.

\subsection{Top-Quark Mass}

A precision determination of the top-quark mass, $M_{\mathrm{t}}$, is an important goal of the Run-II physics program. Since $M_{\mathrm{t}}$ is large, quantum loops involving the top quark are important to include when making SM pre- 


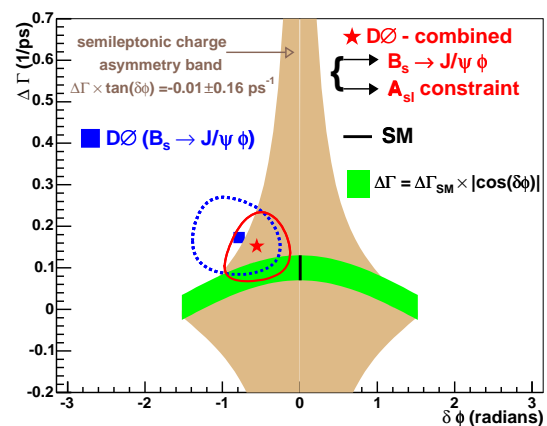

Fig. 8. Combined constraints on $\Delta \Gamma=\Gamma_{H}-\Gamma_{L}$ and $\Phi_{B_{s}}$ from $\mathrm{D} \varnothing$.

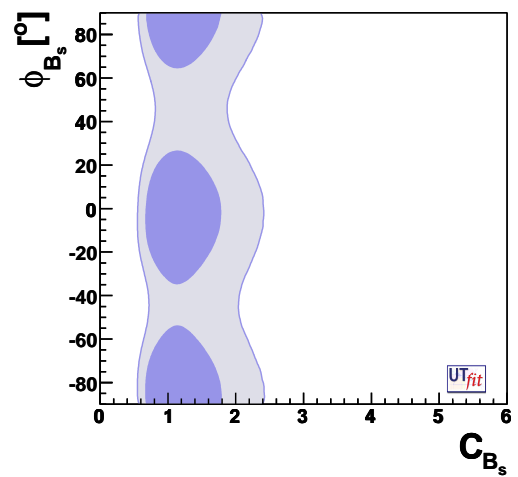

Fig. 9. Combined constraints on New Physics contributions to $\Delta m_{s}$ and the $\mathrm{CP}$ phase $\Phi_{B_{s}}$ using $\mathrm{CDF}$ and $\mathrm{D} \emptyset$ results. The dark (light) areas correspond to the 68 (95)\% C.L. regions. The SM is at $\left(C_{N P}, \Phi_{B_{s}}\right)=\left(1,1^{\circ}\right)$.

dictions of precision electroweak observables. Within the SM it's particularly important to help constrain the mass of the SM higgs boson, $M_{\mathrm{h}}$. It's important to note that the top-quark mass is an important input for any model trying to describe high energy physics. For example, at tree level in the MSSM the lightest higgs mass is constrained to be less than the mass of the $Z$ boson - which has been experimentally excluded. The MSSM is "saved" because loop corrections including the top quark and top squark raise the theoretical upper limit ${ }^{18}$ to about $135 \mathrm{GeV} / c^{2}$, which escapes the present experimental bounds. The magnitude of $M_{\mathrm{t}}$ also impacts soft-SUSY breaking phenomenology and plays a critical role in verifying gauge unification through Renormalization Group Equations.

At the Tevatron top quarks are predominantly produced in $t \bar{t}$ pairs with a crosssection of approximately $7 p b$. Since $M_{\mathrm{t}}>$ $M_{b}+M_{\mathrm{W}}$ and since $V_{t b} \gg V_{t s}, V_{t d}$, the top quark decays before it hadronizes with a branching ratio of $B R\left(t \rightarrow W^{+} b\right) \sim 100 \%$ and the experimental final state is determined by the $W$ decays. Both CDF and $\mathrm{D} \varnothing$ have analyses sensitive to each of the three main final state topologies: dilepton, $t \bar{t} \rightarrow$ $\ell^{+} \nu b \ell^{-} \bar{\nu} \bar{b}$, lepton-plus-jet, $t \bar{t} \rightarrow \ell \nu q q^{\prime} b \bar{b}$, and all-jets, $t \bar{t} \rightarrow q q^{\prime} b q q^{\prime} \bar{b}$. The main features of each of these channels is briefly discussed below. More details can be found in Ref. ${ }^{19}$.

The all-jets (AJT) topology is characterized by a six jet final state and arises when both $W$ s decay hadronically, $W \rightarrow q q^{\prime}$. The principal challenge facing this channel is the suppression of an enormous background from QCD multi-jet production. After all selection requirements, including the identification of both $b$-quark jets in the event using algorithms optimized for $t \bar{t} b$-jets, about 200 $t \bar{t} \rightarrow q q^{\prime} b q q^{\prime} \bar{b}$ events are collected by each experiment in $1 \mathrm{fb}^{-1}$ of data with a purity of about $30 \%$. Since the final state is fully reconstructed for these events kinematic constraints can be employed in order to improve the per-event $M_{\mathrm{t}}$ resolution.

The lepton-plus-jets (LJT) topology arises when one of the $W \mathrm{~s}$ decays hadronically and the other leptonically, $W \rightarrow \ell \nu$. Together with the two $b$-jets from the topquark decays the $W$ decay products produce a final state characterized by one high energy lepton, four jets, and missing momentum from the neutrino. After all selection requirements, including the identification of at least one $b$-jet in the event, about 230 $t \bar{t} \rightarrow \ell \nu q q^{\prime} b \bar{b}$ events are collected by each experiment in $1 \mathrm{fb}^{-1}$ of data with a purity of about $90 \%$. This is the "golden" channel 
since it yields a large sample of $t \bar{t}$ events with small background and still affords the use of kinematic constraints in order to improve the $M_{\mathrm{t}}$ resolution.

The dilepton (DIL) topology arises when both $W$ s decay leptonically and is characterized by two high energy leptons, two jets (both $b$-jets), and large missing momentum due to the two neutrinos. The branching ratio for this final state is about a factor of four smaller than the AJT and LJT topologies. However, the backgrounds can be kept acceptably low without having to require the explicit identification of the $b$-jets. After all selection criteria each experiment collects about $50 t \bar{t} \rightarrow \ell^{+} \nu b \ell^{-} \bar{\nu} \bar{b}$ events in $1 \mathrm{fb}^{-1}$ of data with a purity of about $65 \%$. Owing to the two neutrinos in the final state, this topology is under constrained and the perevent $M_{\mathrm{t}}$ resolution is considerably poorer than for the other two channels.

The analysis methodology is basically the same for all channels and details can be found in these Refs. ${ }^{20}$. After event selection kinematic constraints are applied to determine the best fit top mass per event. The constraints are applied either via a kinematic $\chi^{2}$-fit or more sophisticated matrix element calculations which integrate over initial state unknowns (e.g. parton distribution functions) and experimental resolutions (e.g. jet energies). An ambiguity arises when deciding how to assign the reconstructed jets to the underlying partons from the top-quark and $W$-boson decays. In addition, since at a hadron collider the initial state energy and momentum along the direction of the beamline are unknown, a quadratic ambiguity arises when solving the kinematic constraints for the neutrino momentum. Both of these sources of ambiguity give rise to a combinatoric background which is most (least) severe in the AJT (DIL) channel. For all channels the combinatoric background introduces no bias but does dilute the experimen- tal resolution.

Both CDF and D $\varnothing$ have invested a significant effort in determining their absolute jet energy scale, see for example Ref. ${ }^{21}$. The resulting set of corrections account for non-uniformities and non-linearities in the calorimeter response, and for multipleinteraction, underlying-event, fragmentation and hadronization effects. The corrections are energy and polar-angle dependent and effectively map the detector level jet energy to the underlying parton energy. There are several sources of experimental and modeling uncertainty associated with the corrections. On average they contribute about a $2.5 \%$ uncertainty on the determination of $M_{\mathrm{t}}$ which is a dominant source of systematic uncertainty for both the DIL and AJT channels. Analyses in the LJT channel dramatically reduce this uncertainty by performing an in situ jet energy calibration by comparing the reconstructed $W \rightarrow q q^{\prime}$ mass to the world-average $M_{\mathrm{W}}$. This methodology should also be applicable to the AJT channel, although owing to the additional combinatoric and physics backgrounds present, has not yet been included. This is an important improvement to the methodology since the jet energy scale systematic is now expected to scale with the statistics of the sample for the full Run-II data set, whereas previously it was a systematic uncertainty expected to limit the ultimate precision on $M_{\mathrm{t}}$.

At this conference CDF reported three new results using the full $1 \mathrm{fb}^{-1}$ data set, one in each channel, and $\mathrm{D} \emptyset$ reported an improved analysis result in the DIL channel. More details can be found in Ref. ${ }^{22}$. The CDF and D $\emptyset$ results from Run-I and Run-II are combined to form a world-average top-quark mass. The combination procedure is described in Ref. ${ }^{23}$ and the result is summarized in Fig. 10. The world-average top-quark mass is $M_{\mathrm{t}}=171.4 \pm 1.2$ (sta.) \pm 1.7 (sys.) $\mathrm{GeV} / c^{2}$, corresponding to a total uncertainty of $2.1 \mathrm{GeV} / c^{2}$ and a fractional un- 
certainty of $1.2 \%$. Since the CDF and D $\varnothing$ sensitivities are very similar, this will be further reduced once the $1 \mathrm{fb}^{-1}$ results from $\mathrm{D} \varnothing$ are finalized and included. The ultimate sensitivity of the Tevatron $M_{\mathrm{t}}$ determination is expected to lie in the $<1 \%$ range, far exceeding the Run-II goal and comparable to the ultimate precision presently expected from the LHC experiments ${ }^{24}$. The limitation is a combination of systematic uncertainties associated with signal and background modeling correlated across channels and across experiments (e.g. ISR, FSR, fragmentation, and $q^{2}$ choice). It should be noted that these same uncertainties will also be correlated with the LHC measurements.

There are a couple important caveats which deserve mention here. The interpretation of what, exactly, is measured is complicated by theoretical ambiguities associated with the definition of the mass of a colored object. The cleanest theoretical definition requires a threshold scan of the production cross-section $\sigma\left(e^{+} e^{-} \rightarrow t \bar{t}\right)$ at an ILC. Strictly speaking, the $M_{\mathrm{t}}$ determined here corresponds to a definition specific to the Monte Carlo generators used to calibrate each analysis. These are taken to correspond to the top-quark pole mass, an approxima-

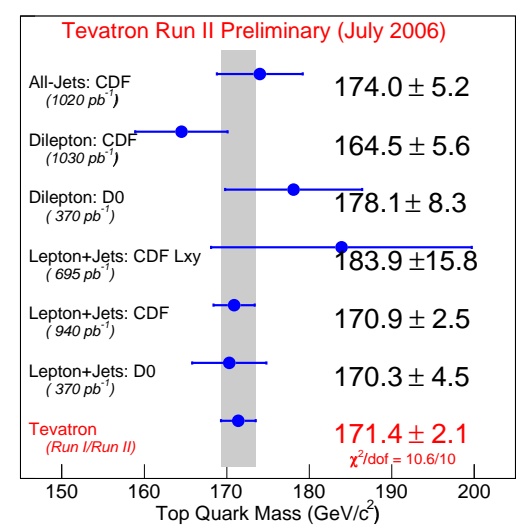

Fig. 10. A summary of the Run-II CDF and D $\varnothing$ $M_{\mathrm{t}}$ measurements used in the combination and the resulting world-average top-quark mass. tion accurate at the level of $<1 \mathrm{GeV} / c^{2}$, well below the present experimental uncertainty and therefore adequately accurate for the time being. However, as the experimental precision improves, additional work will be required to clarify and quantify the relevant theoretical ambiguities. A second important caveat is that systematic uncertainties arising from color-reconnection (CR) effects have not yet been included. While CR models were developed and included in Monte Carlo generators for the $M_{\mathrm{W}}$ measurements made at LEP II, these are not readily useful at a hadron collider since these models must also consistently include the effects of the colorflow from the underlying event (ie. the $p \bar{p}$ beam remnants) and multiple-interactions. Work is ongoing to provide such a model and the corresponding CR uncertainty will be quantified once they are available. It is important to note that both of these caveats also apply to the projected LHC determinations of the top-quark mass.

\subsection{Search for the Higgs Boson}

The CDF and $D \emptyset$ experiments both have vigorous programs searching for new physics arising from a wide array of models. Many of the latest results are summarized in Ref. ${ }^{25}$. I concentrate here on searches for the higgs boson which are discussed in more detail in Ref. ${ }^{26}$.

The higgs field is responsible for generating particle masses and arises in one form or another in many models. In the SM there is a single neutral higgs scalar, $h$, while SUSY models predict five higgs particles, the neutral $h, H, A$, and the charged $H^{ \pm}$. The mass spectra of the SUSY higgs depends on the specifics of the SUSY-breaking parameters (e.g $\tan \beta, m_{0}, m_{1 / 2}$, etc), but in MSSM scenarios the lowest lying higgs always has a mass $<135 \mathrm{GeV} / c^{2}$. As mentioned in Sec. 3.2, in the SM, owing to quantum loop corrections to precision electroweak observ- 
ables, precision $M_{\mathrm{W}}$ and $M_{\mathrm{t}}$ determinations can be used to indirectly constrain the higgsboson mass, $M_{\mathrm{h}}$. The present constraint is shown in Fig. 11 and indicates that a SM higgs is light ${ }^{27}$, where the Tevatron sensitivity is best and the LHC worst.

At the Tevatron the most important SM higgs production mechanisms are $p \bar{p} \rightarrow W h$ and $p \bar{p} \rightarrow Z h$ at low mass $\left(M_{\mathrm{h}}<140 \mathrm{GeV} / c^{2}\right.$ or so), where the branching ratio is dominated by $h \rightarrow b \bar{b}$ and $h \rightarrow \tau^{+} \tau^{-}$. At higher masses, where the decay $h \rightarrow W^{+} W^{-}$begins to dominate, the $g g \rightarrow h$ process also becomes important. The experimental final states are driven by the $W$ and $Z$ decays. Practically, only the $W \rightarrow \ell \nu, Z \rightarrow$ $\ell^{+} \ell^{-}$, and $Z \rightarrow \nu \bar{\nu}$ decays significantly contribute to the search sensitivity since channels involving the the hadronic $W / Z$ decays have to compete with an overwhelming QCD multi-jet background. Although the relative branching ratios of $h \rightarrow b \bar{b}, \tau^{+} \tau^{-}$, and $W^{+} W^{-}$differ in SUSY models (and again depend on the values of the SUSYbreaking parameters) for the most part the most important production mechanisms and final state decays overlap with the SM signatures.

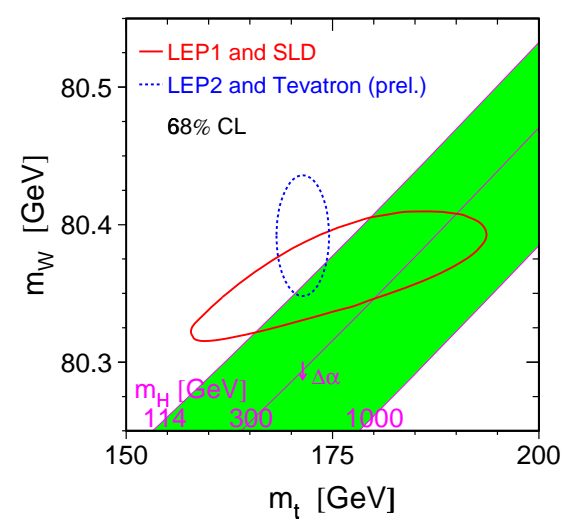

Fig. 11. The indirect constraint on $M_{\mathrm{h}}$ from direct determinations of $M_{\mathrm{W}}$ and $M_{\mathrm{t}}$.
In the SM, the production cross-section is approximately $\sigma(p \bar{p} \rightarrow W h+Z h)=$ $100-300 \mathrm{fb}$ for $M_{\mathrm{h}}=140-110 \mathrm{GeV} / c^{2}$ and $\sigma(g g \rightarrow h)=150-500 f b$ for $M_{\mathrm{h}}=$ $180-140 \mathrm{GeV} / c^{2}$. The SUSY production cross-sections again vary over the SUSYbreaking parameter space, but range from being as small as the SM cross-sections to being significantly larger for $\tan \beta>30$ or so. Due to the SUSY cross-section enhancements CDF and DØ will each have sensitivity for a SUSY higgs boson for all $\tan \beta>30$ and $m_{A}<200 \mathrm{GeV} / c^{2}$ with only $4 \mathrm{fb}^{-1}$ of data.

At this conference both experiments reported new results using the full $1 \mathrm{fb}^{-1}$ data set. The D $\varnothing$ experiment reported new results using the $g g \rightarrow h \rightarrow W^{+} W^{-}$and $p \bar{p} \rightarrow$ $W h \rightarrow W W^{+} W^{-}$topologies important for $M_{\mathrm{h}}>140 \mathrm{GeV} / c^{2}$. The CDF experiment reported new results using the $p \bar{p} \rightarrow W h \rightarrow$ $W b \bar{b}, p \bar{p} \rightarrow Z h \rightarrow Z b \bar{b}$ topologies important for $M_{\mathrm{h}}<140 \mathrm{GeV} / c^{2}$. A final result is obtained by combining about 15 separate CDF and $D \emptyset$ searches that cover all the experimental final states mentioned above. Details of the combination can be found in Ref. ${ }^{28}$. The resulting curve of the production crosssection at the $95 \%$ C.L. upper limit, normalized to the SM expected cross-section, as a function of $M_{\mathrm{h}}$ is shown in Fig. 12. Using only $1 \mathrm{fb}^{-1}$ of data the Tevatron experiments are within a factor of about 7 of the SM at low mass and within a factor of about 3 at high mass. In addition to accumulating another factor of about 7 in data, each experiment expects to further improve the sensitivity of these searches by systematically including analysis improvements across all relevant search channels. Owing to the long lead times involved in properly including new algorithms into an analysis, several improvements have only been included in some of the channels so far. For example, by including improved lepton selections and multi-variate discriminants, the $Z h \rightarrow \ell^{+} \ell^{-} b \bar{b}$ channel achieved an increase in sensitivity equivalent 
to about a 6 times the luminosity. Both experiments are vigorously working to include these and other improvement in all channels. Both CDF and D $\varnothing$ together expect to ultimately reach the required sensitivity to allow a definitive statement about the existence of a SM higgs for all $M_{\mathrm{h}}=110-180 \mathrm{GeV} / c^{2}$ or so.

\section{Conclusions}

The Run-II program at the Fermilab Tevatron began in 2001 and since then has doubled the available data set to both the CDF and $D \emptyset$ experiments each year. It is set to again double the data set in each of the next two years and to deliver a total of $6-8 \mathrm{fb}^{-1}$ per experiment. Most of the results reported at this conference used the full $1 \mathrm{fb}^{-1}$ data set available at the time. The Run-II physics program is an extremely broad one, covering topics in QCD, Electroweak, Bottomand Top-Quark Physics, and Searches for New Phenomena. CDF and D $\varnothing$ have so far each published about 100 physics papers describing these results, which were summarized in over 40 talks at this conference. This past year has been a very successful year for the Tevatron experiments. Highlights include the observation $B_{s}^{0}-\bar{B}_{s}^{0}$ os-

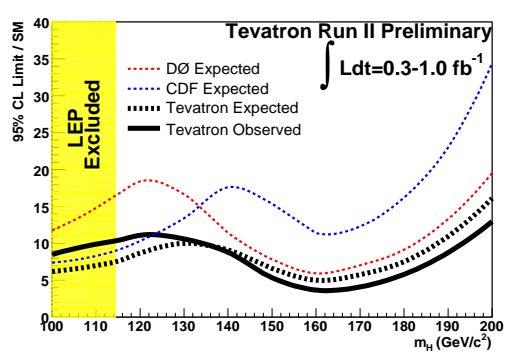

Fig. 12. The Tevatron combined $95 \%$ C.L. upper limit on the SM higgs production cross-section, normalized to the SM expected cross-section, as a function of higgs mass. Differences in the sensitivity of $\mathrm{CDF}$ and $\mathrm{D} \varnothing$ as a function of $M_{\mathrm{h}}$ reflect the differing amounts of data included in the search channels relevant for a given mass range and are not due to any real difference in experimental sensitivity. cillations and the precision determination of $\Delta m_{s}$, which significantly improve the CKM constraints in the $\rho-\eta$ plane by measuring the ratio $\left|V_{t d} / V_{t s}\right|$. The uncertainty on the ratio and resulting CKM constraint is limited by Lattice QCD uncertainties, which are about a factor of 6 larger than the experimental uncertainties. Another highlight is the top-quark mass measurements, which determine $M_{\mathrm{t}}$ with a relative uncertainty of $1.2 \%$, already meeting the Run-II goal. It is expected that ultimately the Tevatron will reach a precision of $<1 \%$ on the top-quark mass, comparable to present expectations for the LHC experiments. At that level of precision additional theoretical ambiguities must be addressed, which will be relevant at both the Tevatron and the LHC. Lastly, impressive progress has been made toward reaching the necessary sensitivity for SM higgs boson searches. Using $1 \mathrm{fb}^{-1}$ of data, the Tevatron combined sensitivity is a factor of 3-7 away from the SM for all $M_{\mathrm{h}}=110-180 \mathrm{GeV} / c^{2}$. Both experiments are vigorously pursuing additional analysis improvements to further enhance the final sensitivity.

The Tevatron program expects to collect another factor of about seven in luminosity for each experiment. I'm confident the LHC will inherit from the Tevatron: a precision determination of $\Delta m_{s}$ and significant constraints on the $\mathrm{CP}$ phase in the $B_{s}$ sector, $M_{\mathrm{t}}\left(\delta M_{\mathrm{t}}<1.5 \mathrm{GeV} / c^{2}\right)$ and $M_{\mathrm{W}}$ $\left(\delta M_{\mathrm{W}}<25 \mathrm{MeV} / c^{2}\right)$ determinations with precisions comparable to those presently predicted for the LHC experiments, a more restricted parameter space for a wide variety of New Physics models, and (with a lot of hard work and some luck) a higgs mass.

\section{Acknowledgments}

The author wishes to thank the organizers for a stimulating conference, to thank the $\mathrm{CDF}$ and $\mathrm{D} \emptyset$ collaborations for trusting him to represent them, and to express 
great gratitude to all those who helped him prepare for the talk including G. Bernardi, G. Borrisov, F. Canelli, G. Gomez-Ceballos, I. Furic, B. Heinemann, A. Hocker, T. Junk, J. Kroll, E. Lipeles, and W. Yao.

\section{References}

1. R. Blair, et al. (CDF Collaboration), The CDF-II Detector Technical Design Report, FERMILAB-PUB-96-390-E (1996); D. Acosta, et al. (CDF Collaboration), Phys. Rev. D 71, 032001 (2005).

2. V.M. Abazov, et al. (DØ Collaboration), Nucl. Instrum. Methods A565, 463 (2006).

3. The parallel speakers were S. Anderson, G. Bernardi, S. Blessing, G. Borrisov, P. Busey, F. Canelli, Y.S. Chung, S. Farrington, R. Field, S. Giagu, I. Gorelov, H. Greenlee, A. Hamilton, K. Hatakeyama, T. Hebbeker, C Hill, A. Hocker, E. Kajfacz, B. Kilminster, A. Kraan, M. Kreps, A. Kupco, M. Lancaster, E. Lipeles, T. Moulik, J. Nielsen, A. Nomerotski, D. O'Neil, L. Pinera, A. Pronko, M. Sanders, P. Savard, C. Schwanenberger, M. Strauss, W. Taylor, M. Verzochi, W. Wagner, D. Wicke, and F. Wuerthwein.

4. In addition to the plenary talk described in this article, see also R. Barlow, Rare $B$ and Tau Decays, E. Gallo, Beyond the Standard Model - Recent Experimental Results, and D. Wood, Precision Electroweak Results, these proceedings.

5. V.M. Abazov, et al. (DØ Collaboration), Phys. Rev. Lett. 97, 021802 (2006); T. Moulik, these proceedings.

6. A. Abulencia, et al. (CDF Collaboration), Phys. Rev. Lett. 97, 062003 (2006); S. Giagu, these proceedings.

7. Charge conjugate modes are implied throughout this article except where explicitly noted.

8. N. Lenardo, Ph.D. Thesis, Massachusetts Institute of Technology, FERMILAB-THESIS2006-18 (2006).

9. H.G. Moser and A. Roussarie, Nucl. Instrum. Methods A384, 491 (1997).

10. S. Eidelman, et al., Phys. Lett. B 592, 1 (2004).

11. M. Okamoto, PoS LAT2005 (2005) 013, hep-lat/0510113.

12. D. Acosta, et al. (CDF Collaboration), Phys. Rev. Lett. 96, 202001 (2006).
13. M. Bona, et al. (UTfit Collaboration), JHEP 0507, 028 (2005); J. Charles, et al. (CKMfitter Collaboration), Eur. Phys. J. C41, 1 (2005).

14. Additional details also available in the articles by M. Bona, S. T'Jampens, and V. Vagnoni, these proceedings.

15. A. Abulencia, et al. (CDF Collaboration), hep-ex/0609040, accepted to Phys. Rev. Lett..

16. G.Borrisov, these proceedings.

17. For additional details see hep-ph/0012219, hep-ph/0406300, hep-ph/0605028 and articles by S. T'Jampens and V. Vagnoni, these proceedings.

18. see for example, S. Heinemeyer, W. Hollik, and G. Weiglein, Eur. Phys. J. C9, 343 (1999) or G. Degrassi, et al., Eur. Phys. J. C28, 133 (2003).

19. C. Hill, and D. O'Neil, these proceedings.

20. A. Abulencia, et al. (CDF Collaboration), Phys. Rev. Lett. 96, 152002 (2006); A. Abulencia, et al. (CDF Collaboration), Phys. Rev. D 73, 032003 (2006); V.M. Abazov, et al. (DØ Collaboration), hep-ex/0609053, submitted to Phys. Rev. D; V.M. Abazov, et al. (DØ Collaboration), hep-ex/0609056, submitted to Phys. Rev. Lett..

21. A. Bhatti, et al., Nucl. Instrum. Methods A566, 375 (2006).

22. F. Canelli, these proceedings.

23. The Tevatron Electroweak Working Group (for the CDF and D $\varnothing$ Collaborations), hep-ex/0608032 (2006).

24. R. Chierici, these proceedings.

25. E. Gallo, these proceedings.

26. B. Kilminster, G. Bernardi, and A. Hocker, these proceedings.

27. C. Parks, these proceedings; the most recent update is available at http://www . cern. ch/LEPEWWG/.

28. The Tevatron New Phenomena and Higgs Working Group (for the CDF and DØ Collaborations), D $\varnothing$ Conf. 5227, CDF Conf. 8384 (2006). 